13

\title{
Датчик слабого магнитного поля на основе азотно-вакансионных центров окраски в кристалле алмаза
}

\author{
(C) А.К. Вершовский, А.К. Дмитриев \\ Физико-технический институт им. А.Ф. Иофрфе РАН, \\ 194021 Санкт-Петербург, Россия \\ e-mail: antver@mail.ioffe.ru
}

Поступило в Редакцию 6 марта 2020 г.

В окончательной редакции 6 марта 2020 г.

Принято к публикации 6 марта 2020 г.

Исследован эффект магнитозависимого поглощения радиочастотного высокочастотного излучения, регистрируемого методом оптически детектируемого магнитного резонанса, и предложено его объяснение. Экспериментально исследован способ высокочастотного возбуждения оптически детектируемого магнитного резонанса в азотно-вакансионных центрах окраски в кристалле алмаза, обеспечивающий магнитометрическую чувствительность на уровне единиц $\mathrm{nT}$ в полосе $1 \mathrm{~Hz}$ в нулевых и слабых $(<0.1 \mathrm{mT})$ магнитных полях. Способ не предполагает использования сверхвысокочастотных полей, что обусловливает его привлекательность для биологических и медицинских применений, в первую очередь — для задач инвазивной магнитоэнцефалографии.

Ключевые слова: оптически детектируемый магнитный резонанс, азотно-вакансионный центр, квантовый магнитометр.

DOI: $10.21883 /$ JTF.2020.08.49547.73-20

\section{Введение}

Естественной областью применения магнитометрических датчиков на азотно-вакансионных центрах окраски (NV-центрах) вследствие их миниатюрности, работоспособности в широком диапазоне температур и полной химической инертности являются биология и медицина. К настоящему времени созданы сотни схем таких датчиков, использующих для измерения индукции магнитного поля эффект зеемановского расщепления линий электронного парамагнитного резонанса. Эти схемы реализованы как на объемных кристаллах, так и на одиночных центрах; разработаны как датчики постоянных полей, так и датчики переменных полей, использующие различные модификации метода спинового эха (обзор этих схем приведен, например, в [1]). Однако почти всем этим датчикам присущи ограничения, препятствующие их использованию именно в медицинских и биологических задачах: во-первых, они используют и соответственно излучают в непосредственной близости к исследуемому объекту интенсивные сверхвысокочастотные (СВЧ) поля; во-вторых, они теряют магнитометрическую чувствительность в слабых $(<0.1 \mathrm{mT})$ магнитных полях, в том числе и в магнитном поле Земли. Это связано как с наличием в решетке алмаза напряжений (strain), которые в этих полях вызывают уширение сигналов оптического детектирования магнитного резонанса (ОДМР), превышающее зеемановское расщепление, так и с перекрытием в слабых полях контуров сигналов, регистрируемых методом ОДМР, и соответствующих переходам между различными спиновыми состояниями. Схемы, не использующие СВЧ поля, но работоспособные только в сильных магнитных полях, рассмотрены в [2-4]. Одновременное устранение обоих ограничивающих факторов при сохранении свойственной сверхкомпактным датчикам на NV-центрах чувствительности могло бы создать условия для их использования в задачах инвазивной магнитоэнцефалографии - такие датчики не имели бы себе равных по чувствительности, достижимой при субмиллиметровых размерах.

В настоящей работе мы приводим результаты исследования эффекта, об обнаружении которого мы впервые сообщили в докладе [5] - эффекта широкополосного (в интервале $1-10 \mathrm{MHz}$ ) магнитозависимого поглощения радиочастотного высокочастотного (ВЧ) излучения, регистрируемого стандартным методом ОДМР, т.е. по уменьшению сигнала фотолюминесценции (эффект ОДМР при ВЧ возбуждении, или ВЧ ОДМР). Мы показываем, что на эффекте ВЧ ОДМР может быть реализован сверхкомпактный датчик слабого магнитного поля, не использующий и не излучающий СВЧ полей. Мы также обсуждаем природу обнаруженного эффекта и предлагаем свое объяснение обнаруженных явлений.

\section{Эксперимент}

Экспериментальная установка была ранее описана в [6,7]; блок-схема установки приведена на рис. 1. Объектом исследования был кристалл алмаза марки SDB1085 60/70 размером $0.3 \times 0.3 \times 0.1 \mathrm{~mm}$ (объем $\left.\sim 0.01 \mathrm{~mm}^{3}\right)$, подвергшийся стандартной процедуре, позволяющей существенно увеличить начальную концентрацию NV-центров - облучению электронами с ин- 


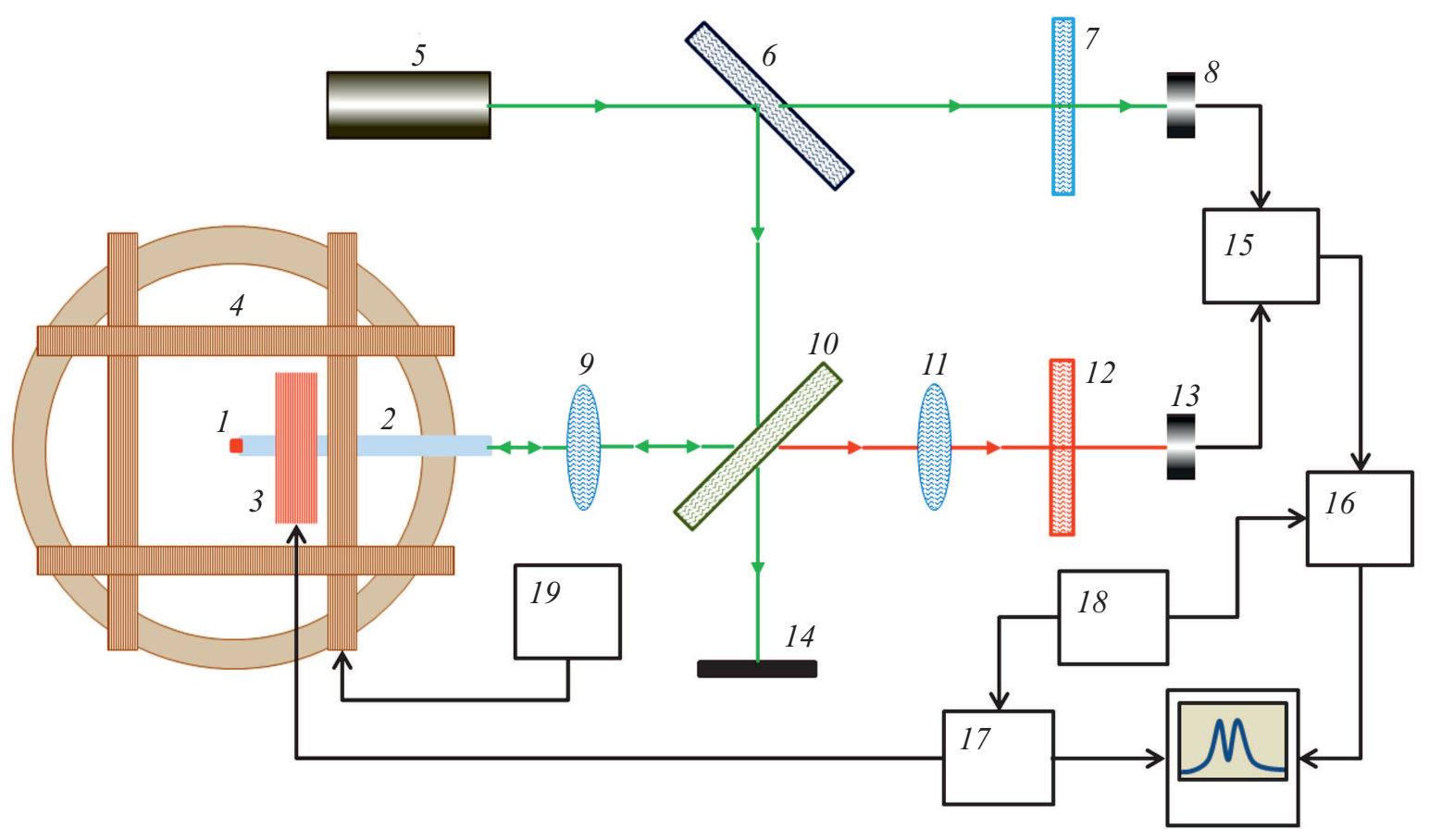

Рис. 1. Блок-схема установки: 1 - кристалл алмаза, $2-$ оптоволокно, 3 - катушка-индуктор ВЧ поля, 4 - кольца Гельмгольца, 5 - лазер, 6 - полупрозрачное зеркало, 7,12 - цветные светофильтры, 8,13 - фотоприемники, 9,11 - фокусирующие линзы, 10 - дихроичное зеркало, 14 - поглотитель лазерного излучения, 15 - балансный (дифференциальный) усилитель, 16 - синхронный детектор, 17 - управляемый ВЧ генератор, 18 - генератор частоты модуляции, 19 - программируемый трехканальный генератор тока.

тенсивностью $\sim 5 \cdot 10^{18} \mathrm{~cm}^{2}$ и отжигу в атмосфере аргона при температуре $800^{\circ} \mathrm{C}$ в течение $2 \mathrm{~h}$. Источником накачки служил твердотельный лазерный модуль с длиной волны излучения $532 \mathrm{~nm}$ и выходной мощностью $15 \mathrm{~mW}$. Излучение накачки передавалось к кристаллу с помощью кварцевого оптоволокна с диаметром центральной части $0.9 \mathrm{~mm}$; это же оптоволокно использовалось для сбора фотолюминесценции на фотоприемник (фотодиод ФД-24К). Волокно с диаметром, существенно большим, чем линейный размер кристалла, было выбрано для увеличения эффективности сбора люминесценции за счет неизбежных потерь интенсивности накачки. Для увеличения эффективности накачки и сбора люминесценции торец оптоволокна вместе с укрепленным на нем кристаллом алмаза был покрыт слоем светоотражающей краски. Свет флюоресценции отделялся от света накачки дихроичным зеркалом Thorlabs DMLP567 и дополнительно красным светофильтром.

Влияние амплитудных флуктуаций лазерного излучения было в значительной мере подавлено за счет использования балансной схемы регистрации сигнала: часть излучения накачки отщеплялась и поступала на дополнительный фотоприемник, после чего интенсивности излучения на двух фотоприемниках выравнивались, и сигналы с них вычитались. Для дополнительного снижения уровня технических шумов мы использовали стопроцентную амплитудную низкочастотную (в диапазоне до сотен Hz) модуляцию ВЧ поля (генератор Rigol 1022) с последующим синхронным детектированием сигнала на частоте модуляции.

Все измерения производились при комнатной темпеpaтуре. ВЧ поля в диапазоне $f_{\mathrm{RF}}=0.1-10 \mathrm{MHz}$ создавались с помощью радиочастотной катушки [7], сигнал на которую подавался с генератора Rigol DG1032Z. Кристалл алмаза был размещен в центре трехкоординатной системы магнитных колец Гельмгольца, напряжение в каждой паре колец создавалось программируемым источником тока Rigol DP832, что позволяло менять направление магнитного поля в телесном угле $4 \pi$, задавая величину его модуля в пределах 0-1 mT.

\section{Результаты эксперимента}

На рис. 2 приведены спектры ОДМР, полученные при ВЧ возбуждении в магнитном поле, направленном вдоль кристаллической оси $\langle 111\rangle$ алмаза, при различных величинах магнитного поля. Видно, что сигналы ОДМР магниточувствительны, причем максимальная чувствительность к изменению магнитного поля достигается в диапазоне частот ВЧ поля $f_{\mathrm{RF}}=4-5 \mathrm{MHz}$. Особенность на частоте $4.95 \mathrm{MHz}$ связана с сигналом резонанса прецессии ядерного спина, соответствующего переходам $|0,0\rangle \leftrightarrow|0, \pm 1\rangle$ (первый индекс обозначает проекцию электронного спина, второй - ядерного); этот сигнал был ранее исследован нами в [8]. Зависимости, аналогичные приведенным на рис. 2, были сняты и для 


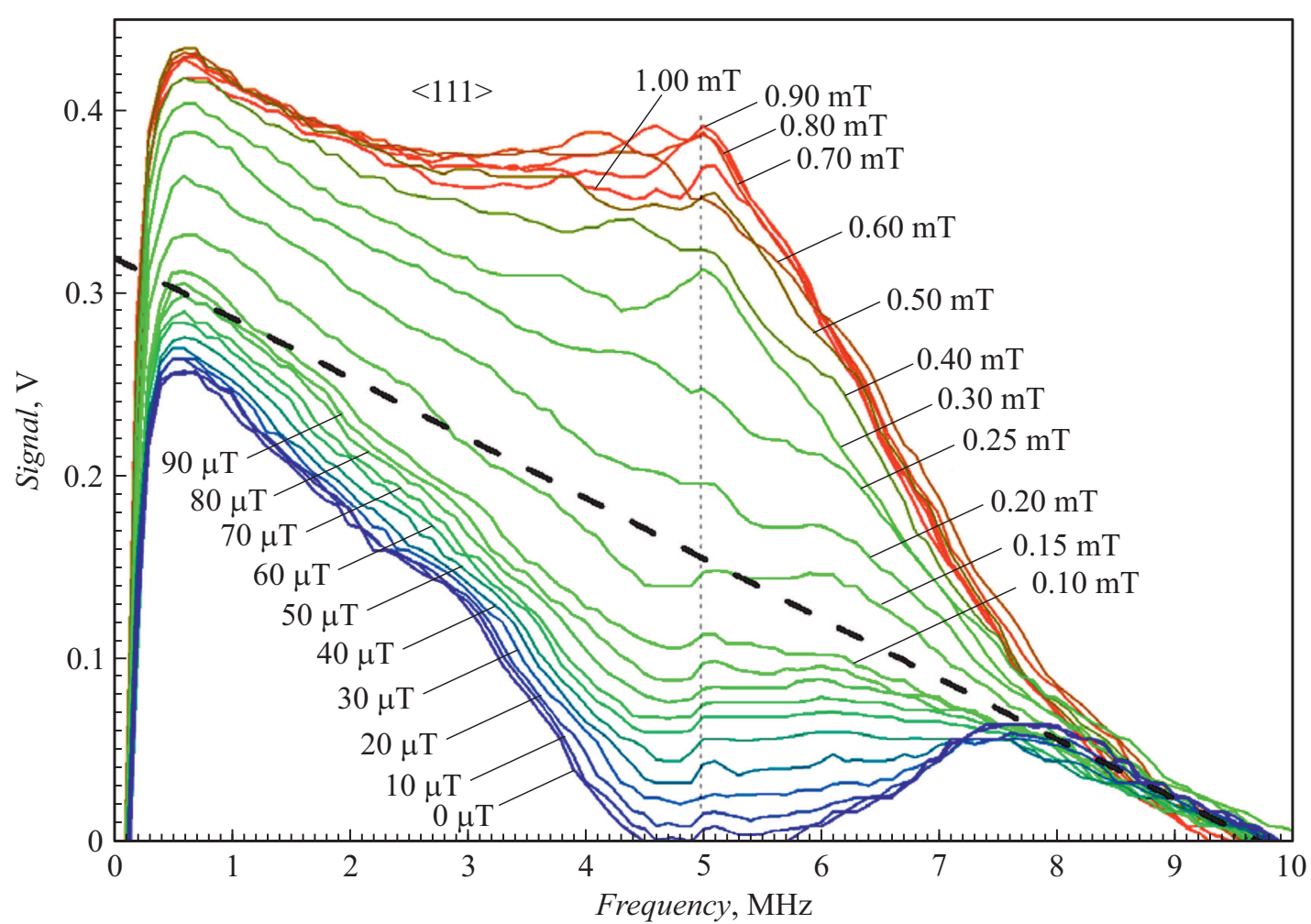

Рис. 2. Спектры радиочастотного ОДМР, полученные в магнитном поле, направленном вдоль кристаллической оси $\langle 111\rangle$ алмаза, при различных величинах индукции магнитного поля.

других направлений внешнего поля; они обнаруживают некоторые различия, но качественно схожи между собой.

На рис. 3 приведены сигналы ОДМР при возбуждении ВЧ полем с частотой $4.5 \mathrm{MHz}$ в зависимости от величины внешнего магнитного поля; разброс экспериментальных данных обусловлен шумом магнитного поля в лаборатории при отсутствии магнитного экранирования. Форма сигналов в первом приближении может быть описана модулем дисперсионной функции $|F(B)|=A \cdot|B| /\left(B_{2}+\Delta^{2}\right)^{2}$, где $\Delta-$ характеристика резонансного контура, наименьшее значение которой $(\Delta=0.52 \mathrm{mT})$ реализуется при направлении внешнего поля В вдоль кристаллографических направлений $\langle 100\rangle$, $\langle 010\rangle,\langle 001\rangle$. При $B \approx 0$ сигнал ОДМР характеризуется резким симметричным провалом, форма и ширина которого очень слабо зависят от направления внешнего магнитного поля. Полуширина резонансного провала, измеренная на половине его глубины, составляет всего $0.087 \mathrm{mT}$, что в сочетании с относительно высоким отношением сигнала к уровню дробового шума позволяет реализовать на нем высокочувствительную скалярную, т. е. чувствительную только к модулю поля, магнитометрическую схему. При этом для повышения линейности отклика в диапазоне $|B|<0.1 \mathrm{mT}$ амплитудная модуляция ВЧ поля может быть заменена низкочастотной модуляцией внешнего поля $B$; при этом частотная зависимость сигнала ОДМР из симметричной преобразуется в антисимметричную с нулем, соответ- ствующим нулевому значению модуля внешнего поля (вставка на рис. 3).

Предельную чувствительность схемы можно оценить, сравнивая крутизну резонанса, т.е. производную амплитуды сигнала ОДМР по величине поля, со среднеквадратичной амплитудой дробового шума фототока в канале регистрации (последняя однозначно определяется величиной фототока). Мы исходим из следующих экспериментальных данных: полный коэффициент преобразования электронного тракта, включающего синхронный детектор, составляет $k=U_{S D} / I_{p h}=1.5 \mathrm{~V} / \mu \mathrm{A}$. Фототок, регистрируемый на сигнальном фотоприемнике, составляет $I_{p h}=40 \mu \mathrm{A}$. Соответствующая среднеквадратичная амплитуда дробового шума равна $I_{S N}=3.6 \mathrm{pA} / \sqrt{\mathrm{Hz}}$, что в пересчете в напряжение на выходе электронного тракта составляет $U_{\mathrm{SN}}=k \cdot I_{S N}=5.4 \mu \mathrm{V} / \sqrt{\mathrm{Hz}}$. Отношение сигнала к дробовому шуму для $B \|\langle 100\rangle$ составляет $\sim 6.4 \cdot 10^{3}$. Максимальная производная сигнала по величине магнитного поля равна $d U_{S D} / d B=1.5 \mathrm{~V} / \mathrm{mT}$. Следовательно, минимальная флуктуация поля $\delta B$, которая может быть разрешена на фоне дробового шума, составляет $\delta B=U_{S N} /\left(d U_{S D} / d B\right)=3.6 \mathrm{nT} / \sqrt{\mathrm{Hz}}$.

Следует отметить, что реальный уровень шума лазерного излучения, регистрируемый в нашем эксперименте, и обусловленный как собственно техническими шумами многомодового твердотельного лазера, так и неидеальностью балансной схемы, превышает уровень 


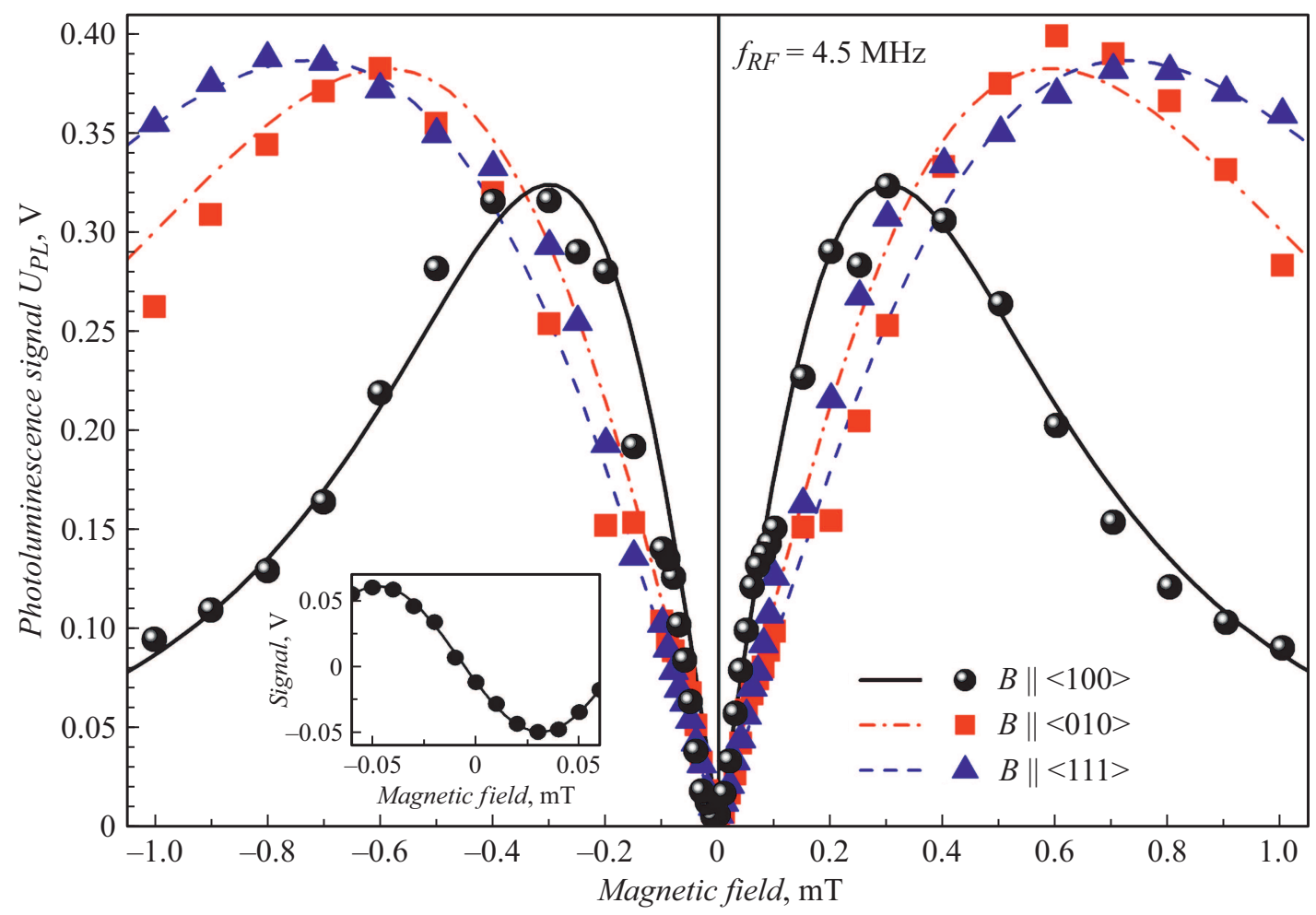

Рис. 3. Сигналы ОДМР при возбуждении амплитудно модулированным ВЧ полем с частотой $4.5 \mathrm{MHz}$ в зависимости от величины внешнего магнитного поля. Внешнее поле приложено в трех направлениях относительно кристаллической решетки. Линии проведены для облегчения восприятия. Вставка - сигнал при $\mathbf{B} \|\langle 100\rangle$ и модуляции магнитного поля.

дробового шума в 4-5 раз. Эти шумы в существенной мере могут быть подавлены при замене твердотельного лазера на диодный лазер с источником тока, охваченным глубокой обратной связью. Дополнительное увеличение чувствительности на порядок и более при данном размере кристалла может быть осуществлено как посредством увеличения мощности накачки (как показано в [9], мощность $15 \mathrm{~mW}$ для кристалла используемых размеров далека от значения насыщения, - даже если не учитывать почти девяностопроцентные потери интенсивности при вводе накачки через оптоволокно, диаметр которого втрое превышает линейный размер кристалла), так и за счет увеличения эффективности сбора фотолюминесценции.

\section{Обсуждение}

Структура уровней NV-центра в алмазе в основном состояния ${ }^{3} \mathrm{~A}_{2}$ с учетом сверхтонкого взаимодействия с ядром азота ${ }^{14} \mathrm{~N}$ изображена на рис. 4; она определяется гамильтонианом $[10,11]$ :

$$
\begin{aligned}
& H=D\left(S_{z}^{2}-\frac{1}{3} \mathbf{S}^{2}\right)+E\left(S_{x}^{2}-S_{y}^{2}\right)+g_{s} \mu_{B} \mathbf{B} \cdot \mathbf{S} \\
& +A_{\|} S_{z} I_{z}+A_{\perp}\left(S_{x} I_{x}+S_{y} I_{y}\right)+P I_{z}^{2}-g_{I} \mu_{N} \mathbf{B} \cdot \mathbf{I},
\end{aligned}
$$

где $\mu_{B}=h \cdot 13.996 \cdot 10^{9} \mathrm{~Hz} / \mathrm{T}$ - магнетон Бора, I спин ядра ${ }^{14} N(I=1), \mathbf{S}$ - электронный спин NV-центра
$(S=1), \quad\left(N=h \cdot 7.622 \cdot 10^{6} \mathrm{~Hz} / \mathrm{T}\right.$ - ядерный магнетон, $D=2.87 \mathrm{GHz}$ и $E$ (порядка нескольких $\mathrm{MHz}$ ) параметры продольного и поперечного расщепления в нулевом поле, $g_{S}=2.003$ и $g_{I}=0.403$ - электронный и ядерный $g$-факторы $[11], A_{\|}=-2.16 \mathrm{MHz}$ и $A_{\perp}=-2.7 \mathrm{MHz}$ - константы продольного и поперечного сверхтонкого расщепления, $P=4.95 \mathrm{MHz}$ - параметр квадрупольного расщепления. В малых $(<1 \mathrm{mT})$ полях поперечное расщепление приводит к возникновению антипересечения уровней $\left|-1, m_{I}\right\rangle$ и $\left|+1, m_{I}\right\rangle$, эффект, всесторонне исследованный в [12]. Смешанные уровни оказываются разделены интервалом $2 E$, в пределах одного кристалла варьирующимся от 0 до (10-12) MHz [13]. Как показано в [13,14], резонансное ВЧ поле может играть роль возмущения, быстро изменяющего энергию системы, и благодаря этому вызывающего запрещенные в стационарном случае переходы между смешанными уровнями (механизм Ландау-Зенера [15]).

Мы считаем, что именно переходы Ландау-Зенера между смешанными уровнями $\left| \pm 1, m_{I}\right\rangle$ определяют наблюдаемые нами спектры ВЧ ОДМР (рис. 2,3). Этот вывод основан на следующих особенностях полученных спектральных зависимостей:

1. Сигнал ВЧ ОДМР регистрируется только в диапазоне частот до $10 \mathrm{MHz}$, что совпадает с шириной распределения расщепления $2 E$ в исследуемом кристалле. 


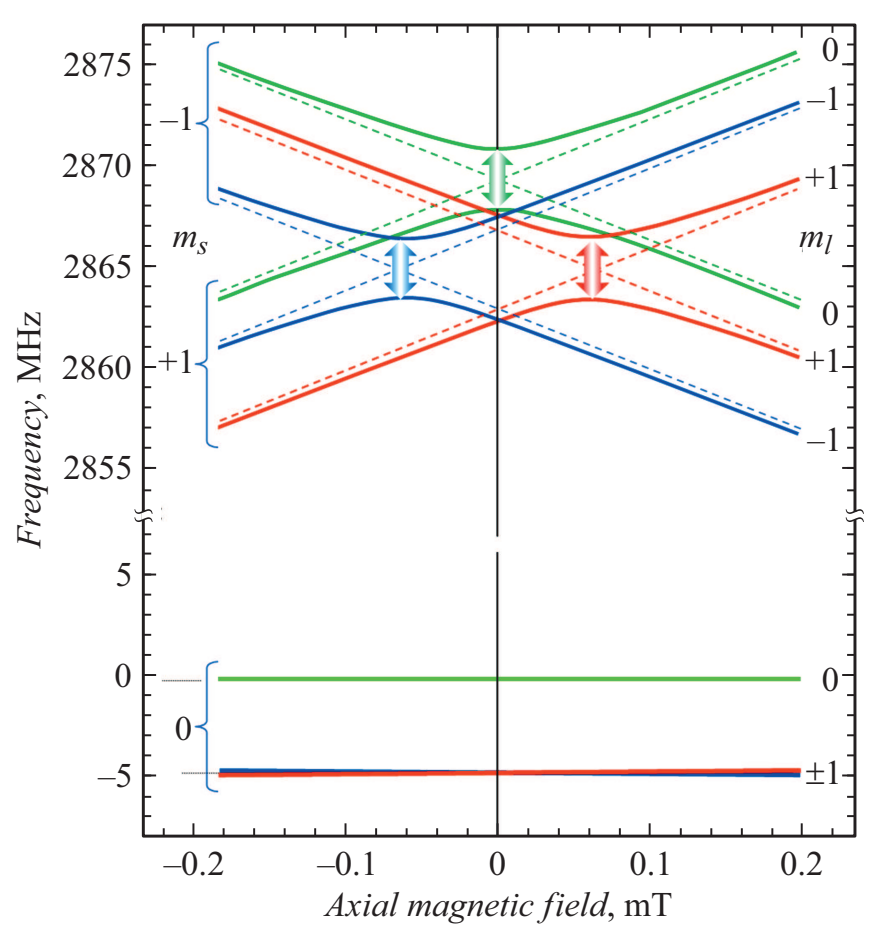

Рис. 4. Схема уровней основного состояния NV-центра в слабых магнитных полях. Чистые состояния изображены штрихом, смешанные - жирными сплошными линиями. Вертикальные стрелки обозначают резонансные радиочастотные поля с частотой $\sim 2 E$, связывающие между собой смешанные состояния.

2. Максимум зависимости от магнитного поля реализуется в интервале частот (4-5) MHz, что хорошо согласуется со средней величиной расщепления $2 E=4.4 \mathrm{MHz}$, измеренной для нашего кристалла в [14].

3. Сигнал ВЧ ОДМР регистрируется только в диапазоне магнитных полей до нескольких $\mathrm{mT}$, что соответствует диапазону антипересечения уровней. Далее, сигнал ВЧ ОДМР симметричен по отношению к величине поля и равен нулю в нулевом поле. Эти особенности можно объяснить, если предположить, что уровни, энергия которых возрастает с модулем поля (например, $|-1,0\rangle$ при $B<0$ и $|+1,0\rangle$ при $B>0$ ), в слабых полях заселены иначе, чем уровни, энергия которых уменьшается с модулем поля (т.е. $|+1,0\rangle$ при $B<0$ и $|-1,0\rangle$ при $B>0)$. Насколько нам известно, этот вопрос пока не исследован ни теоретически, ни экспериментально, но наши данные явным образом свидетельствуют в пользу такого предположения.

\section{Заключение}

Мы исследовали обнаруженный нами эффект слаборезонансного магнитозависимого поглощения ВЧ излучения, регистрируемого методом ОДМР. Мы полагаем, что исследуемый эффект обусловлен эффектом антипересечения уровней $\left|-1, m_{I}\right\rangle$ и $\left|+1, m_{I}\right\rangle$ в нулевых и слабых полях, причем резонансное ВЧ поле вызывает переходы между этими уровнями по механизму ЛандауЗенера. Реализуемые в нашем эксперименте параметры сигнала позволяют реализовать на основе эффекта ВЧ ОДМР скалярный магнитометрический датчик слабого (< $0.1 \mathrm{mT})$ поля, характеризующийся чувствительностью на уровне $3 \mathrm{nT} / \sqrt{\mathrm{Hz}}$ при объеме чувствительного элемента $0.01 \mathrm{~mm}^{3}$, и потенциально способный обеспечить примерно на порядок лучшую чувствительность. При оценке применимости такого датчика к конкретным задачам следует учитывать, что „дальней зоной“, в которой поле магнитного диполя обратно пропорционально кубу расстояния $r$ до него, могут считаться все точки пространства, для которых $r>l$, где $l$ - характерный размер диполя. В случае, когда элементарными источниками магнитного поля являются токи, протекающие в объектах субмиллиметровых размеров (что характерно для задач биофизики и биомедицины), приближение субмиллиметрового датчика на миллиметровое расстояние позволяет существенно увеличить амплитуды измеряемых сигналов и снизить требования к чувствительности датчика - так, датчик на расстоянии $0.1 \mathrm{~mm}$ от активного нейрона может иметь в $10^{6}$ раз худшую чувствительность, чем датчик, находящийся на расстоянии $1 \mathrm{~cm}$. Таким образом, если при исследовании сигналов от поверхностных участков коры головного мозга от датчиков в стандартной магнитоэнцефалографии требуется чувствительность на уровне единиц фемтотесла в полосе $1 \mathrm{~Hz}$ (см., например, [16]), то от инвазивного датчика с характерным линейным размером $0.1 \mathrm{~mm}$ потребуется чувствительность порядка единиц $\mathrm{nT} / \sqrt{\mathrm{Hz}}$. Достижимое при этом пространственное разрешение линейно возрастает с приближением датчика к объекту. Таким образом, предложенная схема может быть использована как в магнитоэнцефалографии, так и в других магнитометрических задачах, требующих компактности датчика и высокого пространственного разрешения.

\section{Финансирование работы}

Исследование выполнено при финансовой поддержке РФФИ в рамках научного проекта № 19-29-10004.

\section{Конфликт интересов}

Авторы заявляют, что у них нет конфликта интересов.

\section{Список литературы}

[1] Abe E., Sasaki K. // J. Appl. Phys. 2018. Vol. 123. N 16. P. 161101.

[2] Simpson D.A., Tetienne J.-P., McCoey J.M., Ganesan K., Hall L.T., Petrou S., Scholten R.E., Hollenberg L.C.L. // Sci. Rep. 2016. Vol. 6. P. 22797. 
[3] Wickenbrock A., Zheng H., Bougas L., Leefer N., Afach S., Jarmola A., Acosta V.M., Budker D. // Appl. Phys. Lett. 2016. Vol. 109. N 5. P. 053505.

[4] Zheng H., Sun Z., Chatzidrosos G., Zhang C., Nakamura K., Sumiya H., Ohshima T., Isoya J., Wrachtrup J., Wickenbrock A., Budker D. // 2019. arXiv:1904.04361.

[5] Dmitriev A.K., Vershovskii A.K. Proc. 7th annual workshop on optically-pumped magnetometers, Mainz, Germany. 14-16 August 2019. C. 105.

[6] Вершовский А.К., Дмитриев А.К. // Письма в ЖТФ. 2015. Т. 41. Вып. 8. С. 78-85.

[7] Dmitriev A.K., Vershovskii A.K. // Appl. Magn. Reson. 2019. Vol. 50. N 4. P. 599-604.

[8] Dmitriev A.K., Vershovskii A.K. // J. Opt. Soc. Am. B. 2016. Vol. 33. N 3. P. B1-B4.

[9] Acosta V.M., Bauch E., Jarmola A., Zipp L.J., Ledbetter M.P., Budker D. // Appl. Phys. Lett. 2010. Vol. 97. N 17. P. 174104.

[10] Felton S., Edmonds A.M., Newton M.E., Martineau P.M., Fisher D., Twitchen D.J., Baker J.M. // Phys. Rev. B. 2009. Vol. 79. N 7. P. 075203.

[11] Fischer R., Jarmola A., Kehayias P., Budker D. // Phys. Rev. B. 2013. Vol. 87. N 12. P. 125207.

[12] Clevenson H., Chen E.H., Dolde F., Teale C., Englund D., Braje D. // Phys. Rev. A. 2016. Vol. 94. N 2. P. 021401.

[13] Fuchs G.D., Burkard G., Klimov P.V., Awschalom D.D. // Nat. Phys. 2011. Vol. 7. N 10. P. 789-793.

[14] Dmitriev A.K., Chen H.Y., Fuchs G.D., Vershovskii A.K. // Phys. Rev. A. 2019. Vol. 100. N 1. P. 011801.

[15] Ди Джкакомо Ф., Никитин Е.Е. // УФН. 2005. Т. 175. Вып. 5. С. 545-547.

[16] Boto E., Meyer S.S., Shah V., Alem O., Knappe S., Kruger P., Fromhold T.M., Lim M., Glover P.M., Morris P.G., Bowtell R., Barnes G.R., Brookes M.J. // NeuroImage. 2017. Vol. 149. P. 404-414. 